\title{
Association of PTPN22 rs2476601 (R620W) polymorphism in Tunisian patients with atopic asthma
}

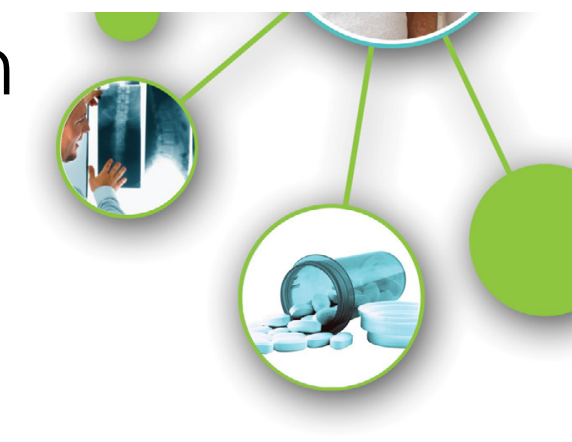

\begin{abstract}
Background: PTPN22 (R620W) gene polymorphism (SNP) gene polymorphism has been associated with diverse inflammatory autoimmune diseases. Only a few studies investigated the influence of PTPN22 (R620W) SNP on atopic asthma risk, severity, and IgE levels.

Methods: Therefore, PTPN22 rs2476601 (R620W) SNP was examined in 171 patients and 323 healthy subjects matched in age, sex and ethnicity.
\end{abstract}

Results: The PTPN22-620*W variant was significantly more prevalent in patients (0.068) than to controls (0.009); $p=1.24 \mathrm{E}-5$, $\mathrm{OR}(95 \% \mathrm{Cl})=7.69$ (3.1-19.07). Analytic results showed that PTPN22*R/W and *W/W genotypes were significantly associated with a family history of atopy, $p=0.014$. Furthermore, asthma control was significantly worse in patients carrying these mutant genotypes, $\mathrm{p}=1.63 \mathrm{E}-7$. Moreover, ${ }^{*} \mathrm{R} / \mathrm{W}$ and ${ }^{*} \mathrm{~W} / \mathrm{W}$ genotypes were significantly correlated to higher levels of total and specific lgE; $p=2.1 \mathrm{E}-3$ and $p=0.037$, respectively. Besides, patients carrying PTPN22-620*R/W and *W/W genotypes had an earlier onset age comparatively to those with ${ }^{*} \mathrm{R} / \mathrm{R}$ genotype but the difference was not significant, $\mathrm{p}=0.061$.

Conclusion: PTPN22 rs2476601 (R620W) polymorphism might influence asthma risk, disease control and IgE synthesis in Tunisian.

Keywords: Atopic, asthma, polymorphism, ptpn22

Abbreviations: Lyp: Lymphoid Protein Tyrosine Phosphatase; PCR: Polymerase Chain Reaction; RFLP PCR: Restricted Fragment-Length Polymorphism; PTPN22: Protein tyrosine phosphatase Non-receptor type 22

\section{Introduction}

Atopic asthma is a chronic inflammatory disorder of the respiratory tract characterized by recurrent episodes of coughing, wheezing and dyspnea and limitation of expiratory airflow [1]. According to the hygiene hypothesis [2], the immunological concept of asthma pathophysiology proposes that a lack of microbial exposure in early childhood leads to a Th2 polarization with reduced regulatory $T$ cell (Treg) activity [3].

PTPN22, called also Lyp (Lymphoid tyrosine phosphatase), plays a regulatory role upon TCR activation [4]. PTPN22 gene exhibits Several Functional Polymorphisms (SNPs) [5]. Among, these SNPs, the rs2476601 SNP is due to a $\mathrm{C} / \mathrm{T}$ nucleotide variation in 1858 position which results in a switch from arginine (R) to tryptophan (W) at position 620 [6]. This
R620W variation impairs PTPN22 interaction with Csk (C-terminal Src Kinase) which results in a significant decrease of $\mathrm{T}$ cell regulation [6]. Several autoimmune diseases including Systemic Lupus Erythematosus, autoimmune diabetes, Graves' disease, rheumatoid arthritis, etc. have been associated with this specific PTPN22 rs2466601 SNP [7]. Nonetheless, few studies investigated its impact on atopic diseases and asthma.

Thus, we aimed to assess the influence of PTPN22 rs2466601 SNP on atopic asthma risk, severity, and IgE levels.

\section{Material and Methods}

\section{Subjects}

This study included 171 patients with atopic asthma and 323 healthy subjects exempt from any atopic disease. Patients have been collected
Tarak Dhaouadi ${ }^{1^{*}}$, Imen

Sfar ${ }^{1}$, Fatma Korbi ${ }^{1}$, Jihen Ben Abdellatif ${ }^{1}$, Ichrak Bannour ${ }^{1,2}$, Hend Bouacha ${ }^{3}$, Raoudha Bousoffara², Taïeb Ben Abdallah ${ }^{1}$, and Yousr Gorgi ${ }^{1}$

\section{${ }^{I}$ Research Laboratory in Immunology of Renal Transplantation and Immunopathology (LRO3SPO1), Charles Nicolle Hospital, Tunis El Manar University, Tunisia \\ 2Pediatrics Department, Tahar Sfar Hospital, Mahdia, Tunisia \\ ${ }^{3}$ Pneumonology Department, Charles Nicolle Hospital, Tunisa, Tunisia \\ *Author for correspondence:}

dhaouaditarak@yahoo.fr 
from pneumonology department of the Charles Nicolle University Hospital in Tunis and pediatrics department of Tahar Sfar Hospital in Mahdia and were diagnosed according to the Global Initiative for Asthma (GINA) guidelines 2006 [1]. Clinical and biological characteristics of the 171 patients are shown in TABLE 1.

Controls were healthy blood donors matched with patients for age, sex, and ethnicity.

Written informed consent to be included in the present study has been obtained from all patients and controls. The Charles Nicolle Hospital's local Ethics committee approved the present study.

\section{Blood sampling and genotyping}

An ordinary salting-out procedure [8,9] have been performed for genomic DNA extraction from EDTA blood.

The PTPN22 rs2476601 polymorphism genotyping was achieved by a restriction fragment length polymorphism-polymerase chain reaction (RFLP-PCR) method. To a $10-\mathrm{uL}$ PCR volume containing $50 \mathrm{ng}$ DNA, 100 pmol of each primer, $1 \mathrm{U}$ of Taq polymerase (Promega, USA), and 0.2 $\mathrm{mmol} / \mathrm{L}$ of each deoxynucleoside triphosphate (dNTP) was added following primers: forward (5'-TGCCCATCCCACACTTTAT-3') and reverse (5'ACCTCCTGGGTTTGTACCTTA-3’). Thermal cycling was performed with an initial activation step at $95^{\circ} \mathrm{C}$ for 15 minutes, 35 cycles of denaturation at $94^{\circ} \mathrm{C}$ for 1 minute, annealing temperature $55^{\circ} \mathrm{C}$ for 1 minute, extension at $72^{\circ} \mathrm{C}$ for 1 minute, and a final extension at $72^{\circ} \mathrm{C}$ for 10 minutes. The PCR product was incubated with $1 \mathrm{U}$ enzyme RsaI (Promega, USA) in a $15-\mu \mathrm{L}$ volume at $37^{\circ} \mathrm{C}$ for 1.5 hours. The PCR generated a 326 bp fragment containing a restriction site for RsaI, which permitted differentiation of the R620- allele (228 bp) and the $620 \mathrm{~W}$-allele $(272 \mathrm{bp})$.

\section{Statistical Analysis}

The results of continuous quantitative variables (Age, Onset Age, and Total IgE level) are expressed as means $\pm \mathrm{SD}$ or median $\left(1^{\text {st }}\right.$ $3^{\text {rd }}$ quartiles), and the means (or median) of groups were compared by ANOVA-test, MannWhitney $\mathrm{U}$ and Kruskal-Wallis, as appropriate (SPSS 11 Inc. Chicago, Illinois, USA). For qualitative variables, the univariable analysis was performed using the chi-square test or Fisher's exact test for small numbers (Epiinfo Stat 7.1 program CDC, Atlanta). Probability (p) values were corrected for the number of tested alleles(pc). p-values $<0.05$ were considered as significant. In order to evaluate the strength of associations, the odds ratios (OR) together with 95\% Confidence Intervals (CI) were calculated.

The Hardy-Weinberg equilibrium was confirmed for the studied SNP via the internet (http://ihg2.helmholtz-muenchen.de/cgi-bin/ hw/hwa1.pl).

\section{Results}

In total, this study included 171 atopic asthma patients and 323 healthy subjects. Mean age of the patients' group was at $24.12 \pm 14.25$ years and the sex-ratio (Men/Women) was 0.78 (75/96). Mean onset-age was at $17.56 \pm$ 14.04 years with $91(53.2 \%)$ patients with an early onset before 16 years old. One hundred and ten (64.3) patients had associated atopic rhinitis and/or conjunctivitis and 61 (35.7\%) had a family history of asthma and/or atopy. Using GINA criteria [1], 174 (86.5\%) patients had controlled asthma, $13(7.6 \%)$ had a partly controlled disease and 10 (5.8\%) had severe uncontrolled asthma. Biologically, total $\mathrm{IgE}$ was $\geq 200 \mathrm{IU} / \mathrm{ml}$ in $90(52.6 \%)$ patients with a median of $239 \mathrm{IU} / \mathrm{ml}$ (59-723). Specific IgE was positive in $116(67.8 \%)$ patients with a median of the level of $17 \mathrm{IU} / \mathrm{ml}$ (0-89) (TABLE 1).

\section{- Analysis of the PTPN22 rs2476601 polymorphism}

The frequency of PTPN22*R/W and *W/W genotypes was significantly higher in patients $(11.11 \%$ and $1.16 \%)$ comparatively controls $(1.86 \%$ and 0$) ; p=1.24 \mathrm{E}-5$, OR $(95 \% \mathrm{CI})=7.39$ (2.92-18.07). Consequently, the PTPN22*W mutant allele was significantly more frequent in patients (0.068) than in controls (0.009); $p=2.8 \mathrm{E}-7$, 7.69 (3.1-19.07) (TABLE 2).

Analysis of PTPN22 SNP with patients features (TABLE 3) showed that those carrying ${ }^{*} \mathrm{R} / \mathrm{W}$ and ${ }^{*} \mathrm{~W} / \mathrm{W}$ genotypes had an earlier onset age (13.71 and 4.58 years) compared to those with the wild ${ }^{*} \mathrm{R} / \mathrm{R}$ genotype $(18.22$ years), but the difference failed to reach significance, $p=0.099$. Accordingly, and even lacking significance, an early onset before 16 years old was more frequent in patients with 


\begin{tabular}{|l|l|}
\hline TABLE 1. Characteristics of atopica asthma patients. \\
\hline Patients & $\mathrm{N}=171$ \\
\hline Age \pm SD (years) & $24.12 \pm 14.25$ \\
\hline Sex ratio (Males / Females) & $0.78(75 / 96)$ \\
\hline Onset age \pm SD (years) & $17.56 \pm 14.05$ \\
\hline Early onset before 16 years old & $91(53.2 \%)$ \\
\hline Exposure to tobacco & $50(29.2 \%)$ \\
\hline Associated atopy (rhinitis and/or conjunctivitis) & $110(64.3 \%)$ \\
\hline Family history of asthma and/or allergy & $61(35.7 \%)$ \\
\hline Asthma classification (GINA 2006) & \\
\hline Controlled & $174(86.5 \%)$ \\
\hline Partly Controlled & $13(7.6 \%)$ \\
\hline Uncontrolled & $10(5.8 \%)$ \\
\hline Total IgE $\geq 200$ IU/ml & $90(52.6 \%)$ \\
\hline Median Total IgE level (1 $1^{\text {st-3rd }}$ quartiles) $(I U / m l)$ & $239(59-723)$ \\
\hline Specific IgE positive & $116(67.8 \%)$ \\
\hline Median Specific lgE level $\left(1^{\text {st }-3^{\text {rd }}}\right.$ quartiles) $(I U / m l)$ & $17(0-89)$ \\
\hline
\end{tabular}

TABLE 2. PTPN22 genotyping results in patients and controls.

\begin{tabular}{|c|c|c|c|c|c|}
\hline \multicolumn{2}{|c|}{ PTPN22 rs2476601 } & Controls $n=323$ & Patients $n=171$ & $p$ & OR (95\% Cl) \\
\hline \multirow{3}{*}{ Genotypes } & $\mathbf{R} / \mathbf{R}$ & 317 (98.14\%) & $150(87.71 \%)$ & \multirow{3}{*}{$1.24 \mathrm{E}-5^{*}$} & \multirow{3}{*}{$7.39 \dagger(2.92-18.07)$} \\
\hline & $R / W$ & $6(1.86 \%)$ & $19(11.11 \%)$ & & \\
\hline & W/W & 0 & $2(1,16 \%)$ & & \\
\hline \multirow{2}{*}{ Alleles } & $\mathbf{R}$ & 0.99 & 0.932 & \multirow{2}{*}{$2.8 \mathrm{E}-7$} & \multirow{2}{*}{$7.69(3.1-19.07)$} \\
\hline & $\mathbf{w}$ & 0.009 & 0.068 & & \\
\hline
\end{tabular}

\section{TABLE 3. Analysis of PTPN22 SNP with clinical and biological features.}

\begin{tabular}{|c|c|c|c|c|}
\hline PTPN22 genotype & $R / R(n=150)$ & $R / W(n=19)$ & $W / W(n=2)$ & $p$ \\
\hline \multicolumn{5}{|l|}{ Gender } \\
\hline Male & $64(85.3 \%)$ & $10(13.3 \%)$ & $1(1.3 \%)$ & \multirow{2}{*}{0.701} \\
\hline Female & $86(89.6 \%)$ & $9(9.4 \%)$ & $1(1 \%)$ & \\
\hline Onset age & 18.22 & 13.71 & 4.58 & 0.099 \\
\hline Early onset before 16 years old & $75(50 \%)$ & $14(73.3 \%)$ & $2(100 \%)$ & 0.061 \\
\hline Associated atopy & $99(66 \%)$ & $10(52.6 \%)$ & $1(50 \%)$ & 0.474 \\
\hline Family history of atopy & $48(32 \%)$ & $11(57.9 \%)$ & $2(100 \%)$ & 0.014 \\
\hline \multicolumn{5}{|l|}{ Asthma classification } \\
\hline Controlled & $137(91.3 \%)$ & $10(52.6 \%)$ & $1(50 \%)$ & \multirow{3}{*}{$1.63 \mathrm{E}-\bar{T}$} \\
\hline Partly controlled & $10(6.7 \%)$ & $3(15.8 \%)$ & 0 & \\
\hline Uncontrolled & $3(2 \%)$ & $6(31.6 \%)$ & $1(50 \%)$ & \\
\hline Total IgE $\geq 200 \mathrm{IU} / \mathrm{ml}$ & $74(49.3 \%)$ & $14(73.7 \%)$ & $2(100 \%)$ & $0.054^{*}$ \\
\hline Total IgE level (IU/ml) & 346.2 & 700.15 & 676.7 & 2.1E-3† \\
\hline Specific IgE positive & $100(66.7 \%)$ & $14(73.7 \%)$ & $2(100 \%)$ & 0.512 \\
\hline Specific IgE level (IU/ml) & 33.01 & 58.09 & 58.63 & 0.037 \\
\hline
\end{tabular}

PTPN22*R/W and *W/W genotypes $(73.3 \%$ and $100 \%)$ than in those carrying the ${ }^{*} \mathrm{R} / \mathrm{R}$ genotype $(50 \%), p=0.061$.

Besides, PTPN22*R/W and *W/W genotypes were significantly associated with a family history of atopy, $p=0.014$ (TABLE 3). Moreover, these PTPN22 mutant genotypes were significantly correlated to more severe asthma with less control, $p=1.63 \mathrm{E}-7$.

Biologically, the presence of total $\mathrm{IgE}$ at a level $\geq 200 \mathrm{IU} / \mathrm{ml}$ was associated with PTPN22*R/W and *W/W genotypes, $p=0.054$.
Subsequently, these PTPN22 mutant genotypes were significantly correlated to higher levels of total and specific IgE; $p=2.1 \mathrm{E}-3$ and $p=0.037$, respectively (TABLE 3).

\section{Discussion}

PTPN22, a negative regulator of TCR, plays a significant role in $\mathrm{T}$ cell regulation upon activation. Thus, functional polymorphisms in the PTPN22 gene could play a significant role in diverse $\mathrm{T}$ cell controlled inflammatory processes such as atopic asthma. 
In the present study, we noted a significant association between the PTPN22*W mutant allele and asthma susceptibility, $p=2.8 \mathrm{E}-7,7.69$ (3.1-19.07). Nonetheless, a previous study in 198 Polish patients with atopic asthma did not show any association with PTPN22 rs2476601 SNP [8]. This discrepancy with our results could be explained by a different genetic background and patients' selection. In fact, if in the present study the majority (86.5\%) of patients had controlled asthma, only $27.27 \%$ had mild asthma in the Polish report [8]. Besides, a study in 112 Chine asthmatic patients with allergic rhinitis showed significant associations of PTPN22 rs1310182 and rs3789604 SNPs, $p=6 \mathrm{E}-6$ and $p=3 \mathrm{E}-6$, respectively [5]. Moreover, a study in West Highland white terriers showed that several PTPN22 polymorphisms play a significant role in atopic dermatitis predisposition.

In this study, the PTPN22 rs2476601 SNP was significantly correlated to a worse control of atopic asthma in patients $(p=1.63 \mathrm{E}-7)$ together with higher levels of both total and specific $\operatorname{IgE}$ $(p=2.1 \mathrm{E}-3$ and $p=0.037)$. These peculiar findings highlight the role of the PTPN22 rs2476601 polymorphism in asthma susceptibility and severity in Tunisian. Nevertheless, these associations of PTPN22 gene rs2476601 SNP requires further replications in other independent cohorts.

\section{Conclusion}

PTPN22 rs2476601 (R620W) polymorphism might influence asthma risk, disease control and IgE synthesis in Tunisian.

\section{Acknowledgment and Funding}

This study was granted and supported by the Research Laboratory in Immunology of Renal Transplantation and Immunopathology (LR03SP01), Charles Nicolle Hospital, Tunis El Manar University, Tunisia.

\section{Competing and Conflicting Interests}

No benefits in any form have been received or will be received from a commercial party related directly or indirectly to the subject of this manuscript.

\section{Author's Contribution}

Professor Yousr Gorgi proposed the study. Imen Sfar realized all the practical part of the study. Tarak Dhaouadi wrote the draft. Tarak Dhaouadi, Imen Sfar, Taieb Ben Abdallah, and Yousr Gorgi analyzed the data. All authors contributed to the design of the study. Yousr Gorgi is the guarantor of the integrity of this study. 


\section{References}

Global Initiative for Asthma. Global strategy for asthma management and prevention. (2016).

Von ME. Allergies, infections and the hygiene hypothesis-the epidemiological evidence. Immunobiology. 212(6), 433439 (2007).

Peters M, Dudziak K, Stiehm M, et al. T-cell polarization depends on the concentration of the danger signal used to activate dendritic cells. Immunol. Cell Biol. 88(5), 537-544 (2010).

Jing L, Ming C, Rong L, et al.
Biochemical and functional studies of lymphoid-specific tyrosine phosphatase (LyP) variants S201F and R266W. PLoS One.7(8), e43631 (2012).

Song SH, Wang XQ, Shen Y, et al. Association between PTPN22/CTLA-4 gene polymorphism and allergic rhinitis with asthma in children. Iran J. Allergy Asthma Immunol. 15(5), 413-419 (2016).

Gianchecchi E, Palombi M, Fierabracci A. The putative role of the C1858T polymorphism of protein tyrosine phosphatase PTPN22 gene in autoimmunity. Autoimmun Rev. 12(7), 717-725 (2013).
Vang T, Miletic AV, Bottini N, et al. Protein tyrosine phosphatase PTPN22 in human autoimmunity. Autoimmunity. 40(6), 453-456 (2007).

Majorczyk E, Jasek M, Ploski R, et al. Association of PTPN22 single nucleotide polymorphism with rheumatoid arthritis but not with allergic asthma. Eur. J. Hum. Genet. 15(10), 1043-1048 (2017).

Roque JB, O'Leary CA, Kyaw-Tanner $\mathrm{M}$, et al. PTPN22 polymorphisms indicate a role for this gene in atopic dermatitis in West Highland white terriers. BMC Research Notes. 4(1), 571 (2011). 